\title{
Questions and Controversies in the Clinical Application of Tyrosine Kinase Inhibitors to Treat Patients with Radioiodine-Refractory Differentiated Thyroid Carcinoma: Expert Perspectives
}

\section{(이요 $\odot$}

\section{Authors}

Frederik A. Verburg1, 2, Holger Amthauer ${ }^{3}$, Ina Binse ${ }^{4 \dagger}$, Ingo Brink ${ }^{5}$, Andreas Buck ${ }^{6}$, Andreas Darr ${ }^{7}$, Christine Dierks ${ }^{8}$, Christine Koch ${ }^{9}$, Ute König ${ }^{10}$, Michael C. Kreiss| ${ }^{11}$, Markus Luster ${ }^{1}$, Christoph Reuter ${ }^{12}$, Klemens Scheidhauer ${ }^{13}$, Holger Sven Willenberg ${ }^{14}\left(\mathbb{D}\right.$, Andreas Zielke ${ }^{15}$, Matthias Schott ${ }^{16}$

Affiliations

1 Department of Nuclear Medicine, University Hospital Marburg, Marburg, Germany

2 Erasmus Medical Center, Department of Radiology and Nuclear Medicine, Rotterdam, The Netherlands

3 Charité - Universitätsmedizin Berlin, Freie Universität Berlin, Humboldt-Universität zu Berlin, and Berlin Institute of Health, Department of Nuclear Medicine, Berlin, Germany

4 Department of Nuclear Medicine, University Clinic Essen, Essen, Germany

5 Department of Medical Diagnostics and Therapy, Ernst von Bergmann Hospital Potsdam, Potsdam, Germany

6 Department of Nuclear Medicine, University Hospital Würzburg, Würzburg, Germany

7 Department of Nuclear Medicine, University Hospital Jena, Jena, Germany

8 Department of Medical Oncology, University Hospital Freiburg, Freiburg, Germany

9 Department of Gastroenterology, Hepatology, and Endocrinology, University Clinic Frankfurt, Frankfurt Am Main, Germany

10 Department of Gastroenterology and Endocrinology, University of Göttingen, Göttingen, Germany

11 Division of Nuclear Medicine, Department of Radiology and Nuclear Medicine, Otto von Guericke University Magdeburg, Magdeburg, Germany

12 Department of Palliative Care, Hannover Medical School, Hannover, Germany

13 Interdisclipinary Endocrine Center, Technical University of Munich, Munich, Germany

14 Division of Endocrinology and Metabolism, Medical University of Rostock, Rostock, Germany

15 Department of Endocrine Surgery, Diakonie Klinikum Stuttgart, Stuttgart, Germany

16 Division of Endocrinology, University Hospital Düsseldorf, Düsseldorf, Germany

$\dagger \quad$ In memoriam. This paper is dedicated to the memory of our colleague, Dr. Ina Binse, who passed away during the development of the manuscript.
Key words

multi-kinase inhibitors, treatment initiation and discontinuation, dosing, second-line therapy, lenvatinib, sorafenib

received 26.04.2020

accepted after revision $\quad 26.01 .2021$

\section{Bibliography}

Horm Metab Res 2021; 53: 149-160

DOI 10.1055/a-1380-4154

ISSN 0018-5043

(C) 2021. The Author(s).

This is an open access article published by Thieme under the terms of the Creative Commons Attribution-NonDerivative-NonCommercial-License, permitting copying and reproduction so long as the original work is given appropriate credit. Contents may not be used for commecial purposes, or adapted, remixed, transformed or built upon. (https://creativecommons.org/ licenses/by-nc-nd/4.0/)

Georg Thieme Verlag KG, Rüdigerstraße 14,

70469 Stuttgart, Germany

Correspondence

Frederik A. Verburg M.D., PhD.

Department of Radiology and Nuclear Medicine

Erasmus Medical Center

Doctor Molewaterplein 40

3015 GD Rotterdam

The Netherlands

Tel.: + 31107040704 ,

f.verburg@erasmusmc.nl

$\oplus$ Supplementary material is available under https://doi. org/10.1055/a-1380-4154.

\section{ABSTRACT}

Notwithstanding regulatory approval of lenvatinib and sorafenib to treat radioiodine-refractory differentiated thyroid carcinoma (RAI-R DTC), important questions and controversies persist regarding this use of these tyrosine kinase inhibitors (TKIs). RAI-R DTC experts from German tertiary referral centers convened to identify and explore such issues; this paper summarizes their discussions. One challenge is determining when 
to start TKI therapy. Decision-making should be shared between patients and multidisciplinary caregivers, and should consider tumor size/burden, growth rate, and site(s), the key drivers of RAI-R DTC morbidity and mortality, along with current and projected tumor-related symptomatology, co-morbidities, and performance status. Another question involves choice of first-line TKIs. Currently, lenvatinib is generally preferred, due to greater increase in progression-free survival versus placebo treatment and higher response rate in its pivotal trial versus that of sorafenib; additionally, in those studies, lenvatinib but not sorafenib showed overall survival benefit in subgroup analysis. Whether recommended maximum or lower TKI starting doses better balance anti-tumor effects versus tolerability is also unresolved. Exploratory analyses of lenvatinib pivotal study data suggest dose-response effects, possibly favoring higher dosing; however, results are awaited of a prospective comparison of lenvatinib starting regimens. Some controversy surrounds determination of net therapeutic benefit, the key criterion for continuing TKI therapy: if tolerability is acceptable, overall disease control may justify further treatment despite limited but manageable progression. Future research should assess potential guideposts for starting TKIs; fine-tune dosing strategies and further characterize antitumor efficacy; and evaluate interventions to prevent and/or treat TKI toxicity, particularly palmar-plantar erythrodysesthesia and fatigue.

\section{Introduction}

Although its definition remains somewhat controversial [1, 2], radioiodine-refractory differentiated thyroid carcinoma (RAI-R DTC) may be described as DTC that either has lost, or never had, ability to take up radioiodine in some or all lesions, or that fails to show an adequate response to radioiodine therapy [3]; the key molecular mechanism is decrease or loss in expression of the sodium iodide symporter, which is responsible for ${ }^{131}{ }^{1}$ uptake [4].

Absence of radioiodine uptake generally is discerned, and clinically relevant, only when RAI-R DTC is non-resectable, hence individuals with this condition will typically display distant metastases at diagnosis. Because to date, radioiodine remains the most effective, and only potentially curative, systemic therapy for DTC, RAI-R DTC has had a poor prognosis: with historic conventional treatment strategies, 10-year survival can be as low as $10 \%$ [4].

Tyrosine kinase inhibitors (TKIs) are a relatively new class of small-molecule drugs that have demonstrated considerable anti-tumor efficacy against RAI-R DTC [4-6]: in prospective, randomized, placebo-controlled phase 3 clinical trials, single-agent therapy with the TKIs sorafenib (Nexavar, Bayer, Berlin, Germany) or lenvatinib (Lenvima, Eisai, Tokyo, Japan) significantly increased progression-free survival (PFS) in patients with such disease [7, 8]. Additionally, lenvatinib significantly improved overall survival in the subgroup of patients $>65$ years old [9]. These observations led regulatory authorities in the European Union, the United States, and elsewhere to approve these drugs as treatments for RAI-R DTC $[10,11]$.

Notwithstanding these developments, challenges persist regarding the use of TKIs in RAI-R DTC. These include limited ability to develop strongly evidence-based standardized paradigms for using these agents. This limited ability stems from the rarity of RAI-R DTC, which comprises only an approximately $5 \%$ "subfraction" of DTC cases, and from the considerable heterogeneity in clinical presentation and course among this small patient population [4]. Other challenges in TKI therapy include the frequent, and sometimes substantial toxicity of these agents [12-14].

To help address these challenges, several panels of RAI-R DTC experts from German tertiary referral centers recently convened to identify and explore key questions and controversies regarding TKI use in this setting. This paper summarizes the panels' perspec- tives on these issues and, where applicable, reviews supporting data for these viewpoints. These data include published evidence as well as the panelists' "real-world" clinical experience. Unless noted otherwise, suggestions and commentary made herein represent panelist opinion.

\section{Criteria for starting TKI therapy in patients with RAI-R DTC}

As alluded to earlier, the natural history of RAI-R DTC may range from an indolent, asymptomatic course over months or even years, to rapid progression with major morbidity, for example, one or more of pathological fracture, spinal cord compression, dyspnea on exertion, or cachexia [15]. These symptoms often result in substantial quality-of-life impairment, and are sometimes life-threatening.

Due to the variable clinical course of RAI-R DTC and the toxicity, side effects, and relatively high acquisition costs of TKIs, it can be difficult to determine when to start these drugs. The most important concept in this process is shared decision-making between the patient and the multidisciplinary team that should take sustained responsibility for the management of his or her care. This team should comprise knowledgeable specialists in this rare and complex condition $[3,16]$.

A variety of criteria have been proposed for when to consider starting TKIs ( $\triangleright$ Table 1 ). Tumor size/burden, growth rate, and site(s), the key drivers of RAI-R DTC morbidity and mortality [15], are the principal clinical factors to take into account, along with the patient's current and projected tumor-related symptomatology, co-morbidities, and performance status [3]. There is broad agreement that symptomatic disease or potentially imminently symptomatic disease, for example, progressing lesions near critical anatomic structures including the esophagus, the trachea, or the blood vessels of the neck, are an indication for TKIs $[3,15,17,18]$. Indeed, some panelists argued that appearance of symptoms, for example, pain or respiratory distress, may reflect too long a delay in starting such therapy. Brain metastasis also may be an indication for this intervention, although TKI efficacy against such lesions has not yet been established [17].

Tuttle et al. [15] have suggested integrating tumor size/burden, growth rate, and site(s) to identify each patient's individual “inflec- 
- Table 1 Potential criteria to consider starting TKI therapy of radioiodine-refractory DTC.

\section{Source}

Lenvatinib SELECT [8] or sorafenib DECISION [7] pivotal trials

Tuttle et al “inflection point” [15]

ATA 2015 clinical practice guideline for management of adult thyroid nodules and DTC [3]

NCCN clinical practice guidelines: thyroid carcinoma [17]
Criterion/criteria to consider starting TKI therapy

- $\geq 20 \%$ increase in sum of longest diameters of target lesions defined by RECIST [19] within prior $12 \pm 1$ months (SELECT) or 14 months (DECISION) OR

- Cumulative activity $>22 \mathrm{GBq}(600 \mathrm{mCi})^{131}$ I + radiologic evidence of progression within 13 months (SELECT)

- Tumor diameter doubling time $<1$ year $+\sim 1.0 \mathrm{~cm}$ metastatic focus OR

- Tumor diameter doubling time $1-2$ years $+\sim 1.0-1.5 \mathrm{~cm}$ metastatic focus OR

- Tumor diameter doubling time 2-4 years $+1.5-2.0 \mathrm{~cm}$ metastatic focus OR

- Symptomatic disease OR

- Risk of imminently symptomatic disease due to lesion location near critical anatomic structures or due to large tumor burden comprising high number of small lesions

- Rapidly progressive, symptomatic and/or imminently (i.e., in <6 months) threatening disease ${ }^{a}$

- Progressive and/or symptomatic disease

- Central nervous system metastases

ATA: American Thyroid Association; NCCN: National Comprehensive Cancer Network; RECIST: Response Evaluation Criteria in Solid Tumors;

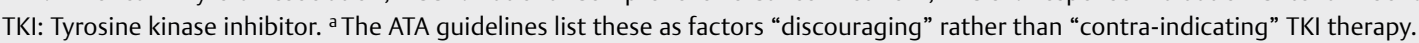

tion point" regarding the course and management of RAI-R DTC. The inflection point is defined as the earliest time when RAI-R DTC "structural disease progression" becomes "clinically significant" and when TKI therapy should be contemplated in asymptomatic patients. Arguing that tumor size follows an exponential growth curve with notable consistency over the course of RAI-R DTC, Tuttle et al. focus on the structural disease progression rate in the form of tumor diameter doubling time. This variable is calculated based on serial cross-sectional imaging and Response Evaluation Criteria in Solid Tumors (RECIST) [19]. The authors opine that a patient's overall clinical course likely will be more accurately prognosticated if in the serial images, the summed diameters of (the same) multiple metastases are compared. The authors suggest two online resources that may assist in the calculation:

- www.thyroid.org/professionals/calcu;ators/thyroidwith-nodules

- www.kuma-h.or.jp/english/about/doubling-time-progressioncalculator/(both last accessed 28 October 2020).

Most panelists agreed that tumor diameter doubling time may be a more reliable prognostic factor than is thyroglobulin doubling time. Although thyroglobulin is undoubtedly useful, interpretation of serial determinations of this analyte in patients with RAI-R DTC may be confounded by the divergence between thyroglobulin synthesis and cell growth/proliferation. Another potential confounding factor is decrease or loss of thyroglobulin expression because of tumor de-differentiation that may occur in this late-stage disease setting [20]. Hence in RAI-R DTC, it is important to consider tumor growth via serial cross-sectional imaging, in addition to, or even instead of, through a biochemical biomarker.

The metabolic activity of the tumor may provide another potentially useful and direct input regarding disease progression in RAI-R DTC. This variable may be gauged via comparison of tumoral standardized uptake value(s), i.e., of semi-quantitative measure- ments of the intensity of radiotracer uptake, on serial fluorodeoxygluxose PET [21]. Some panelists felt that such comparison might be especially helpful when serum thyroglobulin measures $\geq 2-3 \mu \mathrm{g} / \mathrm{l}$.

The panel opined that the "inflection point" concept could be useful. However, when applied clinically, the concept needs to be supplemented with additional considerations. For example, the patient's views regarding treatment need to be taken into account, for example, whether he or she prefers a "minimalist" or "maximalist" approach to systemic therapy, or something in between. The patient's underlying condition, for example, comorbidities, should factor into the decision-making: indeed, the 2015 American Thyroid Association guidelines for the treatment of adult DTC [3] list as factors "discouraging" TKI therapy a number of conditions that potentially could decrease tolerability, or increase risk or severity of side effects of TKIs ( $\triangleright$ Table 2). Besides comorbidities, Eastern Cooperative Oncology Group (ECOG) performance status [22] is another variable reflecting the patient's general condition that provides useful data for decision-making on whether and when to start TKI therapy of RAI-R DTC. However, the panelists discussed that ECOG performance status has the disadvantage of being a subjective and somewhat "fuzzy" variable. Moreover, the fact that ECOG performance status may be assessed either by the treating physician, a nurse, or the patient himself or herself might pose inter-test and intra-test reliability and reproducibility issues. It may well be worth investigating whether more objective measures, for example, blood pressure after 100 steps, or the patient-reported European Organization for Research and Treatment of Cancer QLQ-C30 quality-of-life instrument (https://www.eortc.org/app/uploads/ sites/2/2018/02/SCmanual.pdf, last accessed 28 October 2020) would have more predictive and prognostic value than does ECOG performance status.

Regarding using the patient's overall health status to guide decision-making on when to start TKI therapy, it is notable that in contrast to the Phase 3 studies, some analyses [23-26] of "real-world" 
$\checkmark$ Table 2 Suggested contra-indications to TKI therapy of RAI-R DTC.

\begin{tabular}{|c|c|}
\hline Source & Suggested contra-indication(s) \\
\hline $\begin{array}{l}\text { Lenvatinib and sorafenib } \\
\text { European product labeling } \\
\text { [10-11] }\end{array}$ & $\begin{array}{l}\text { - Hypersensitivity to active drug or excipients } \\
\text { - Breast-feeding (lenvatinib) }\end{array}$ \\
\hline $\begin{array}{l}\text { Lenvatinib SELECT [8] pivotal trial } \\
\text { exclusion criteriaa }\end{array}$ & $\begin{array}{l}\text { - Proteinuria } \geq 1 \mathrm{~g} / 24 \mathrm{~h} \\
\text { - Significant cardiovascular or gastrointestinal dysfunction }\end{array}$ \\
\hline $\begin{array}{l}\text { Sorafenib DECISION [7] exclusion } \\
\text { criteriaa[67] }\end{array}$ & $\begin{array}{l}\text { " Major surgery, open biopsy, significant traumatic injury within } \leq 30 \text { days } \\
\text { " Non-healing wound, ulcer, bone fracture or grade } \geq 2 \text { infection } \\
\text { - Grade } 3 \text { hemorrhage or bleeding event within } \leq 3 \text { months or evidence or history of bleeding diathesis or } \\
\text { coagulopathy or tracheal, bronchial, or esophageal infiltration with significant risk of bleeding } \\
\text { - Clinically significant cardiac disease and/or uncontrolled hypertension }(>150 / 90 \mathrm{mmHg}) \text { despite optimal therapy }\end{array}$ \\
\hline Tuttle et al “inflection point” [15] & $\begin{array}{l}\text { - Asymptomatic metastases in non-critical locations with tumor diameter doubling time }>4 \text { years (criterion to } \\
\text { consider active surveillance) }\end{array}$ \\
\hline $\begin{array}{l}\text { ATA } 2015 \text { clinical practice } \\
\text { guideline for management of } \\
\text { adult thyroid nodules and DTC } \\
{[3]^{\mathrm{b}}}\end{array}$ & $\begin{array}{l}\text { - Active/recent intestinal disease } \\
\text { " Liver disease } \\
\text { " Recent bleeding or coagulopathy } \\
\text { " Cachexia, low weight, or poor nutrition } \\
\text { " Poorly-controlled hypertension } \\
\text { " Prolonged QTc interval/history of significant arrhythmia } \\
\text { " Recent suicidal ideation } \\
\text { " Due to other comorbidities, life expectancy "too brief to justify systemic therapy" } \\
\text { " Recent tracheal radiation therapy } \\
\text { " Untreated brain metastases (controversial) }\end{array}$ \\
\hline $\begin{array}{l}\text { NCCN clinical practice guidelines: } \\
\text { thyroid carcinoma [17] }\end{array}$ & $\begin{array}{l}\text { " Asymptomatic stable or slowly progressing indolent disease assuming no brain metastasis (situation in which } \\
\text { active surveillance is "often appropriate") }\end{array}$ \\
\hline
\end{tabular}

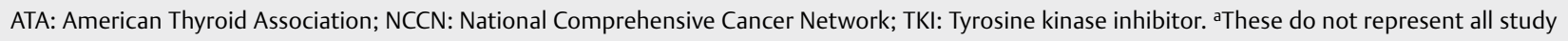

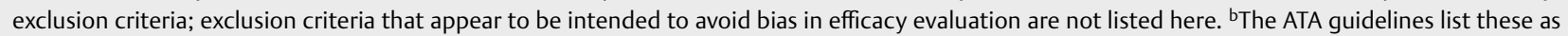
factors "discouraging" rather than "contra-indicating" TKI therapy.

experience with TKIs in patients in a "discouraging” state of health, have found decreased, albeit still substantial, efficacy against RAI-R DTC. The patients included in these "real-world" analyses tended to have more advanced disease and heavier pre-treatment compared to the Phase 3 study samples. Indeed, a small $(n=13)$ single-center study of lenvatinib [27], in which all patients were ECOG 0-1 and TKI-naïve, registered the longest median progression-free survival, 22 [95\% confidence interval (CI) 14-35] months of any "real-world" experience yet published. Some investigators, as well as some panelists, have argued that these observations support the concept that TKI therapy should be started when patients still have limited tumor burden and good general condition [23].

Younger patient age also arguably may mandate a quicker "trigger" to start TKI therapy of RAI-R DTC. The rationale for this view is that manifestation of such disease earlier in life may reflect more aggressive tumor that should be addressed sooner.

\section{Active surveillance protocols for patients not yet on TKIs}

Optimal timing of follow-up testing in patients with RAI-R DTC who are under active surveillance before starting a TKI remains an open issue. Until data are available comparing outcomes of patients on different active surveillance protocols, a prudent approach may be to let monitoring intervals be dictated by the patient's clinical pic- ture, site(s) of metastases, and tumor diameter and thyroglobulin velocities. For example, since patients suffering from metastatic spread confined to the lungs often show few if any symptoms and comparatively slow progression, lung-only metastases may allow longer intervals between monitoring visits than do loco-regional (i.e., cervical) recurrence or bone metastasis. If thyroglobulin is $>1 \mu \mathrm{g} /$ l but relatively stable, active surveillance testing might take place either every 3-6 months or every 6-9 months, absent clinical or radiological factors indicating shorter intervals.

\section{Choice of TKls}

In most countries, the choice of TKIs for RAI-R DTC until recently has been between the only two agents that had been approved to treat this disease, lenvatinib and sorafenib. These multi-kinase inhibitors remain the main options to treat RAI-R DTC, except in the case of specific mutations (see below). Lenvatinib and sorafenib have overlapping as well as distinct molecular targets [4]. Both selectively inhibit vascular endothelial growth factor receptors 1-3, platelet-derived growth factor receptor $\alpha$, and RET proto-oncogene, albeit via distinct modes of binding [28]; lenvatinib also selectively inhibits fibroblast growth factor receptors 1-4 and KIT proto-oncogene receptor tyrosine kinase, while sorafenib also selectively inhibits platelet-derived growth factor receptor $\beta$ and RAF. 
However, in everyday practice, the choice between lenvatinib and sorafenib is made based on clinical rather than molecular factors. Importantly, no trial directly comparing the pair yet has been published, so clinicians must rely on other evidence, expert opinion, and patient preference in making this choice.

In the panelists' opinion and in most cases in current everyday practice, lenvatinib is considered the first-line agent, and it is designated as "preferred" in the August 2020 United States National Comprehensive Cancer Network (NCCN) thyroid carcinoma treatment guidelines [17].

There are four main rationales for the preference for lenvatinib. First, compared with placebo in their respective Phase III trials in RAI-R DTC, SELECT [8], and DECISION [7], lenvatinib was associated with greater absolute and relative increases in median PFS than was sorafenib [7, 8]; PFS was the primary endpoint of both studies. For lenvatinib, the median PFS associated with active treatment was 18.3 months, versus 3.6 months for placebo, an absolute difference of 14.7 months and a relative difference of just over $400 \%$ [8]. For sorafenib, the corresponding values were 10.8 months versus 5.8 months, an absolute difference of 5.0 months and a relative difference of $86 \%$ [7]. Interestingly, the impressive improvement in PFS with lenvatinib was achieved even though the lenvatinib study sample had an appreciable percentage of patients with TKI pretreatment $(25 \%, 66 / 261$, in the lenvatinib arm and $21 \%, 27 / 131$, in the placebo arm). Also of note, a recent systematic review and network meta-analysis of randomized controlled trials of the two TKIs in advanced and/or metastatic DTC [29] suggested greater efficacy for lenvatinib.

Second, the literature contains some evidence [9] of an active treatment-associated gain in overall survival in SELECT [8], but not in DECISION [7]. Namely, in a pre-specified subgroup analysis, patients $>65$ years old who received lenvatinib $(n=106)$ had a hazard ratio $(\mathrm{HR})(95 \% \mathrm{Cl})$ of $0.53(0.31-0.91)$ for mortality relative to their counterparts given placebo $(n=50)(p=0.02)$. For both lenvatinib and sorafenib, however, the crossover design of the pivotal studies, in which patients received active agent after progressing on placebo, may have obscured detection of survival benefits of the TKI versus placebo overall or in subgroups.

Third, in the respective pivotal trials in RAI-R DTC, lenvatinib had a much higher objective (complete + partial) response rate than did sorafenib, $65 \%$ (169/261) [8] versus $12 \%(24 / 196)$ [7].

Lastly, there is a common clinical impression that the side effects of lenvatinib may tend to be more tolerable and manageable than are those of sorafenib; albeit both agents have frequent and sometimes important toxicities. This perspective may be provocatively phrased as boiling down to a question of addressing hypertension versus palmar-plantar erythrodysesthesia (hand-foot syndrome). Aligning with this impression was the finding in a recent meta-analysis of patients with advanced DTC, RAI-R DTC, or both by Yu et al. [13]. In this meta-analysis, sorafenib was associated with a higher rate of adverse events requiring treatment discontinuation than was lenvatinib: $18.1 \%$ (42/238) versus $12.8 \%$ (53/419). However, this difference did not attain statistical significance; the odds ratio $(95 \% \mathrm{Cl})$ for toxicity prompting treatment cessation was $1.52(0.98-2.36), p=0.06$, in sorafenib versus lenvatinib. The Yu et al meta-analysis included 2 sorafenib studies $(N=238)$ and 5 lenvatinib studies $(n=419)$.
As has been noted, though, the perceived differences between the TKIs in PFS and overall survival, objective response rate, and toxicity, are not based on a prospective, randomized, controlled, study directly comparing the two drugs, which to date, has not been performed; indeed, the drugs' respective pivotal trials [7, 30] substantially differed in inclusion/exclusion criteria and the characteristics of their study samples. In particular, any perceived difference in overall safety outcomes at this point must be regarded as anecdotal.

\section{Starting regimen of TKls}

The starting regimen of lenvatinib or sorafenib that optimally balances efficacy versus toxicity is another unresolved issue in the TKI therapy of RAI-R DTC: is it best to begin with the recommended starting dose and to decrease if warranted by adverse events, or to begin with a lower dose and if that dose is tolerated, to escalate to a possibly more efficacious level?

Two factors account for the uncertainty regarding initial dosing. First, treatment-related toxicity frequently led to a changed regimen in the active agent arms of the SELECT and DECISION trials, both of which used the doses currently recommended in product labeling. The respective incidences of toxicity-related treatment interruption were 82.4 and $66.2 \%$, of toxicity-related dose reduction, 67.8 and $64.3 \%$, and of toxicity-related treatment discontinuation, 14.2 and $18.8 \%$, for lenvatinib [8] and sorafenib [7], respectively. Second, to date, no study comparing safety, tolerability, and efficacy of different starting doses of the respective TKI has been published. However, at least one such trial is underway for lenvatinib in RAI-R DTC: the E7080-G000-211 randomized, double-blind, multicenter Phase II study (identifier NCT02702388; https://clinicaltrials.gov/ct2/show/NCT02702388, last accessed 28 October 2020 ) is comparing a starting dose of $18 \mathrm{mg}$ daily versus the currently-recommended $24 \mathrm{mg}$ daily dose.

So far, evidence regarding lenvatinib therapy of RAI-R DTC appears to favor higher starting doses. Perhaps most notably, two exploratory analyses [31,32] of the SELECT study data suggest a cumulative dose-response effect with the drug. A post hoc analysis of the relationship to PFS of the length of cumulative treatment interruptions due to adverse events [32] found that patients in the active treatment arm who had shorter cumulative interruptions (total $<10 \%$ of treatment duration; median 19 days; $n=134$ ) had better median PFS than did their counterparts with longer cumulative interruptions (total $\geq 10 \%$ of treatment duration; median 61 days, $n=127)$ : not reached versus 12.8 ( $95 \% \mathrm{Cl}$ 9.3-16.5) months, after a 17.1-month median follow-up. Additionally, in a multivariate analysis also accounting for age group, gender, region, race, body-mass index, and ECOG performance status category, dose interruption was the only variable other than performance status category that was independently associated with PFS: $\mathrm{HR}(95 \% \mathrm{Cl})$ for progression, shorter versus longer interruption: $0.47,95 \% \mathrm{Cl} 0.31-$ 0.71 , nominal $p<0.001$. Further, patients with shorter interruptions had higher objective response rates: $76 \%$ (102/134) versus $53 \%(67 / 127)$. The SELECT protocol called for dose interruption to allow grade 3 or intolerable grade 2 adverse events to resolve to grade $0-1$ or to baseline severity. Patients in the "shorter interruption" subgroup had a median lenvatinib dose intensity of $20.1 \mathrm{mg} /$ 
day, $84 \%$ of the planned level, versus $14.6 \mathrm{mg} /$ day, $61 \%$ of the planned level, for the "longer interruption" subgroup.

A second exploratory analysis of SELECT data [31], focusing on tumor size changes over time, showed that increased lenvatinib exposure, reflected by area under the curve, correlated $\left(R^{2}=0.355\right)$ with greater tumor size reduction in the first 8 weeks of treatment. This was the period with the most pronounced reduction in tumor size in the overall active treatment group. Additionally, increased lenvatinib treatment duration correlated with tumor size decrease ( $R^{2}$ not reported), albeit this finding may have been at least partly attributable to patients receiving the TKI for a longer time because they had a better response.

Arguably somewhat aligned with observations linking treatment intensity and efficacy were findings in a 78-patient single-center analysis from the University of Pisa (including 15 SELECT patients) [33]. In that analysis, occurrence of treatment-related hypertension, nausea/ anorexia, or weight loss of any grade was significantly associated with longer median PFS, 25 months versus 7 months, $p=0.02$ for hypertension, and 45 months versus 7 months, $p<0.001$ for the other two adverse reaction types. Presumably, toxicity was related to greater treatment intensity, and that, in turn, to efficacy. In SELECT, no tested adverse event was associated with the primary endpoint, PFS [12,34]. Nonetheless, in multivariate analyses, presence of diarrhea [12] or hypertension [34] did appear to be related to longer overall survival. However, some investigators have argued that toxicity should not be considered a surrogate measure of clinical benefit [35].

Regarding TKI starting doses, it also should be noted that a case report involving lenvatinib [36] and a retrospective analysis [37] and small prospective study involving sorafenib [38] all suggest that good antitumor efficacy can be seen when doses are lower than recommended levels. A recent analysis of Taiwanese "real-world" experience of sorafenib treatment of progressive advanced DTC ( $n=36, n=34$ with RAI-R DTC) [39] concluded that starting from half-dose may minimize incidence of high-grade toxicities, but that higher maintenance dose ( $\geq 600 \mathrm{mg}$ daily) was associated with longer PFS.

It is hoped that the results of the lenvatinib comparative dosing study will provide important insight regarding the question of TKI starting doses, at least for this agent. Also of interest, and worthy of further investigation, is the concept of plasma [40] or serum [41] TKI levels serving as a guide for titrating a patient's treatment dose to improve efficacy and tolerability; analyses of clinical trial samples in patients with thyroid cancer [42] or hepatocellular carcinoma [43] given lenvatinib, and a case history [44] of a patient with RAI-R DTC given sorafenib suggest that this approach may be helpful.

\section{Response to and discontinuation of TKI treatment}

Three aspects of response of RAI-R DTC to TKI therapy are well-accepted. First, although this therapy is associated with statistically, and, more importantly, clinically significant benefit regarding PFS $[7,8]$ and possibly, overall survival $[9]$ in many patients with RAI-R DTC, the modality is not curative in this setting [17]. Second, structural responses to TKIs should be defined by RECIST criteria [19], based on serial computed tomography or magnetic resonance imaging, as should disease stability. Third, at least with lenvatinib, tumor response, when it occurs, tends to be most marked during the first 8 weeks of treatment, but continues, albeit at a slower pace, during subsequent therapy [31].

The principal open issues related to response to TKls surround the determination of net therapeutic benefit in particular patients. This concept has been reasonably suggested as the ultimate criterion for continuing TKI therapy [3], and hinges on the anti-disease efficacy, toxicity and tolerability, and quality-of-life effects of the TKI in the given individual. In particular, in certain cases, presence of progressive disease arguably should not be equated with absence of efficacy and benefit. These cases include patients developing slow and/or limited progression after a good general or local tumor response, especially those with only local progression or a small number of new lesions that can be handled by directed therapies $[3,18]$. In such patients, the TKI may be controlling the overall disease, notwithstanding limited, and, importantly, manageable progression. A decrease in the rate of progression even has been proposed as sufficing to justify continuation of treatment with a given TKI, if that decrease would be substantial enough to lengthen survival [15]; this somewhat controversial argument also might apply if tumor symptomatology would be decreased, or the development of symptoms deferred, despite progression, to a sufficient extent that quality-of-life would be materially better than that in the absence of TKI therapy.

\section{Second-line TKI therapy}

Evidence is clear $[8,26,45-48]$ that many patients discontinuing a first-line TKI due to disease progression, treatment-related toxicity, or both, may benefit clinically from a second-line TKI. However, when to begin a second-line (or later-line) TKI is unclear. A pragmatic approach might be to base the start time of the new TKI on the reason for discontinuation of the first-line agent, and on the patient's general condition. If first-line TKI therapy has been ended due to disease progression, consideration should be given to immediately starting a second-line TKI, especially if the first-line agent was well-tolerated. However, if the discontinued first-line TKI was associated with a decline in ECOG performance status, or, especially, substantial weight/body mass loss [49], a brief pause between first-line and second-line therapy may help ameliorate the patient's nutrition, and with it, general condition. Such a pause may be especially appropriate when disease progression is relatively indolent, or does not affect critical/vulnerable anatomic sites.

If the first-line TKI has been discontinued due to poor tolerability, despite a tumor response or stable disease, second-line TKI therapy generally should be withheld until severe or clinically meaningful adverse events have resolved, or at least, markedly improved; some panelists believe that waiting 2-3 half-lives of the first TKI may be another possible strategy. To guide the starting time of second-line therapy when the first-line TKI is discontinued for poor tolerability, it would be of interest to have data regarding how long disease response persists after lenvatinib or sorafenib discontinuation. To date, such data have not been published.

In the panelists' opinion, two alternatives to second-line TKI therapy might be considered in specific scenarios. When a patient has been on less than the recommended dose of the first-line TKI, for example, when he or she has been given $14 \mathrm{mg}$ daily rather than $24 \mathrm{mg}$ daily of lenvatinib, an increased dosage of the first-line agent might be tried before that agent is discontinued in favor of a sec- 
ond-line TKI. Also, if an individual with previous disease control with a first-line TKI has had a "drug holiday" of several months due to toxicity or patient preference, the first-line agent might be re-attempted before a second-line TKI is tried. Additionally, a case report [48] recently was published of successful re-challenge with lenvatinib in an elderly female with inoperable metastatic papillary thyroid cancer, who had had progression after initial response to first-line lenvatinib and second-line sorafenib. It should be noted that the above-described approaches are empirical and outside of product labeling, and currently supported by very limited published evidence.

\section{Adjunctive treatment for patients on TKIs}

To date, little if any literature has appeared regarding adjunctive treatment for patients on TKIs. In our (anecdotal) experience, resection of small numbers of lymph node metastases or isolated progressive metastases in other sites in otherwise stable patients may help control local symptoms or focal progression. However, more major metastectomy is probably contra-indicated in most cases, due to the potential negative effects of TKIs on wound healing $[50,51]$.

Some patients receiving TKIs for progressive RAI-R DTC also have subtypes of metastases that continue to respond to radioiodine ("mixed" disease with radioiodine-avid as well as radioiodine non-avid lesions). So far, no reports have been published about the effects on occasional radioiodine administration in addition to TKI. However, ${ }^{131}$ I appears to have largely non-overlapping toxicities with those of TKIs, and observations of some authors suggest that this combination can be administered safely in selected cases. In theory, combining external beam radiotherapy (EBRT) with a TKI may also provide palliation.

Nonetheless, the rationale for giving radioiodine therapy, EBRT, or both to patients receiving TKI treatment of RAI-R DTC should be clarified, given the potential additive toxicity [52] and the lack thus far of documented benefits regarding PFS, overall survival, or quality-of-life. Also, it should be noted that use of either of these TKIs in combination therapy of RAI-R DTC remains outside of lenvatinib or sorafenib product labeling.

\section{TKI-related adverse events (AEs)}

The toxicities of sorafenib and lenvatinib are generally well-known, with a predictable pattern and timing [51]. Although there is overlap in their adverse events profiles, specific toxicities appear to differ in incidence between the drugs. The meta-analysis by Yu et al. [13] found that among any-grade toxicities, hypertension, nausea, vomiting, and voice change were more commonly noted in patients given lenvatinib, while palmar-plantar erythrodysesthesia, rash, hypocalcemia, and elevated liver enzymes were more frequently seen among those receiving sorafenib. Among more severe, that is, grade $\geq 3$ toxicities, weight loss, hypertension, and nausea were reported more often in patients on lenvatinib, while palmar-plantar erythrodysesthesia, hypocalcemia, and elevated alanine aminotransferase concentration were more commonly observed in patients given sorafenib [13]. Adverse reactions may occur more frequently in older than in younger patients; an analysis of experience in SELECT showed that the treatment subgroup $>65$ years old (median age: 71 years) had an $89 \%$ rate of any Grade $\geq 3$ toxicity
(94/106 patients) compared to a $67 \%$ rate $(104 / 155$ patients) in their counterparts $\leq 65$ years old (median age: 56 years), $p<0.001$ [9]. TKI side effects profiles in patients with RAI-R DTC also may differ according to patients' ethnicity [53].

According to the literature, unwanted reactions to TKIs tend to develop quickly during the first weeks or even days of treatment [35]; however, in "real-world" practice, where there is less intense follow-up than in clinical trials, initial observation of certain adverse events may occur later. Some toxicities may be self-limiting and diminish over time; however, it is unclear to what extent this observation is an artifact of patients learning to better cope with the side effect(s) and hence reporting them less frequently [12].

Perhaps the most important open questions regarding sorafenib and lenvatinib safety and tolerability relate to which measures to deploy in preventing and treating drug-related adverse events. Beyond the dose interruption/reduction and treatment discontinuation schemes delineated in the drugs' product labeling $[10,11]$, a number of interventions are used for these purposes in everyday practice ( $\triangleright$ Table 3 ). However, the benefit of these interventions tends to be anecdotal: few if any formal studies have characterized or compared the efficacy of these measures. Additionally, interventions to directly or sufficiently address some TKI side effects, perhaps most notably fatigue [35] or palmar-plantar erythrodysesthesia [54], currently are lacking.

To optimize TKI tolerability, one prime goal is to identify potentially serious adverse reactions that initially are not symptomatic, for example, hepatotoxicity, prolonged QTC, and cardiotoxicity, particularly hypertension, before they become so [3]. Another prime goal is to identify and treat rapidly symptomatic toxicities while they still are at a lesser grade of severity, since doing so presumably lessens morbidity and eases and speeds recovery [35].

Key to achieving both goals is a skilled, attentive multidisciplinary team, including nurses and pharmacists [55] as well as physicians, and including well-informed and engaged patients and significant others [35]. Tools such as diaries or smartphone applications to monitor toxicities can be helpful in maximizing patients' and significant others' contribution to this team effort [35].

\section{Future Directions}

As alluded to throughout earlier sections of this paper, numerous "open issues" remain regarding use of TKIs in RAI-R DTC. Investigation should focus on at least five main areas. First, the prognostic and predictive values of variables potentially useful in determining if and when to start TKIs should be assessed. For example, data from SELECT [8] and DECISION [7] could be analyzed post hoc to determine the association of tumor diameter doubling time at the start of TKI therapy with response to such treatment, with PFS, and with overall survival. Such research would test the clinical utility of the Tuttle et al. disease "inflection point" model [15] for starting TKIs. As noted earlier, the value of variables that may be more precise and/or objective than is ECOG performance status in reflecting the patient's overall condition should be assessed.

Second, more research should be carried out regarding TKI dosing. Besides the optimal starting dose of lenvatinib or sorafenib, the correlation between plasma or serum drug levels and response to therapy and well as frequency and intensity of side effects [42-44] 


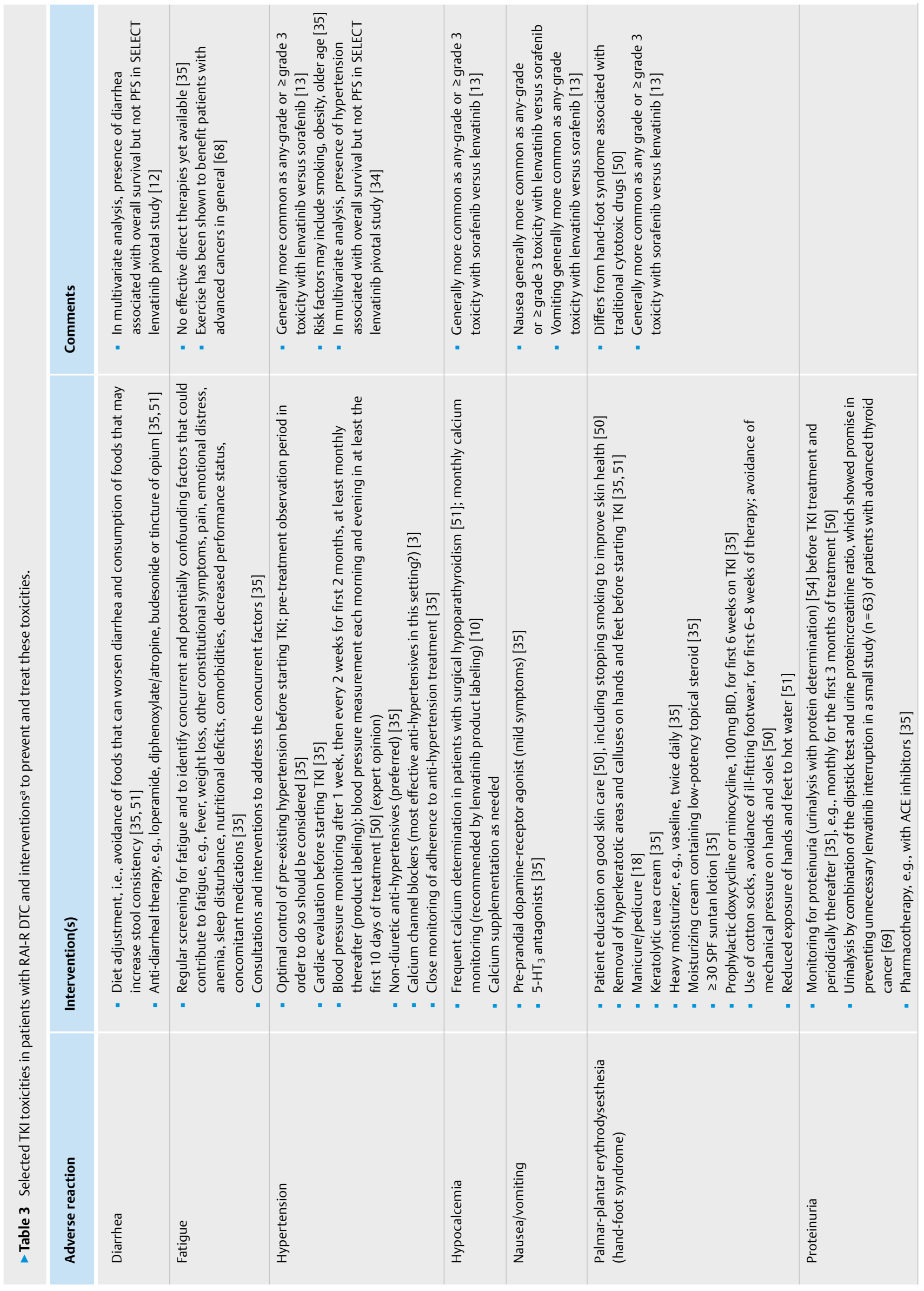




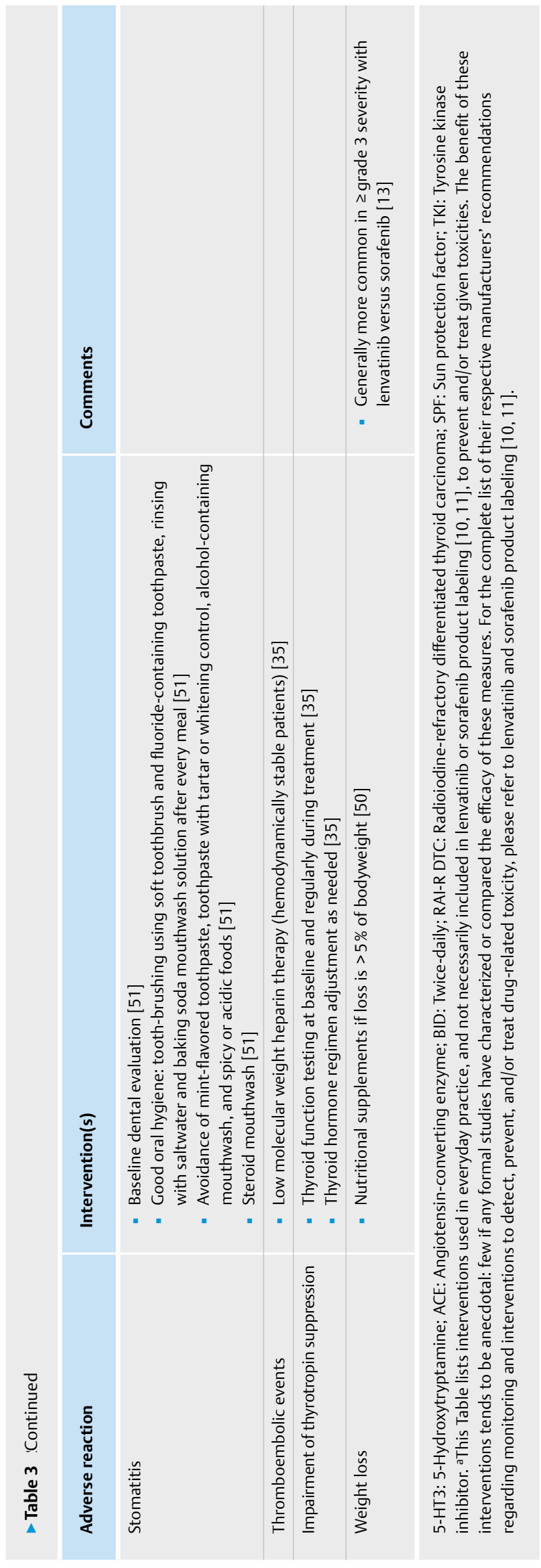

need to be studied. Additionally, the achievable dose intensity and outcomes of "on/off" schedules, for example, therapy followed by rest for a week each, represents an intriguing topic for investigation.

Third, retrospective analyses and prospective studies should be carried out regarding additional facets of TKI efficacy in RAI-R DTC. As noted above, these could include examining the duration of tumor responses after these agents are discontinued due to toxicity or patient preference, and the ability of lenvatinib or sorafenib to prevent appearance of new metastases. Too, it may be informative to calculate outcomes of active treatment in SELECT [8] and DECISION [7] when patients with RECIST stable disease are included alongside partial and complete RECIST responders in a "tumor control" subgroup. Formal study of response to central nervous system metastases to TKIs also could be fruitful.

Fourth, interventions to prevent and/or manage TKI toxicity also should undergo formal evaluation. Palmar-plantar erythrodysethesia and fatigue should be high-priority targets of such work.

Lastly, use of other agents in combinations with TKIs [56, 57] should be studied. Related to this topic would be improved classification of RAI-R DTC, with more nuanced assessment of radioiodine scintigraphy findings and of response to radioiodine therapy than is now frequently performed in everyday practice when identifying DTC as RAI-R [2]. For example, degree of radioiodine uptake on scintigraphy should be evaluated in light of the ${ }^{131}$ I activity, image acquisition protocol, and timing post-radioiodine administration of the scan; response to radioiodine therapy should be evaluated in light of the size and interval of the ${ }^{131}$ I administrations and the duration of progression-free survival post-therapy. Along these lines, retrospective analyses correlating RAI scintigraphic and therapeutic variables with response of particular lesions to TKIs might be of interest. Likewise, prospective study of TKI-radioiodine combination therapy may be warranted in carefully-selected cases.

Besides ${ }^{131} \mathrm{I}$, intriguing candidates for use along with TKIs in RAI-R DTC include potential re-differentiation agents, for example, 13-cis-retinoic acid or 11-cis-retinol plus radioiodine therapy, vinorelbine [58], paclitaxel plus radiation therapy [59], vemurafenib for patients with $B R A F$ mutation [60,61], and checkpoint inhibitors such as pembrolizumab (Keytruda, Merck, Kenilworth, NJ, USA), which have undergone study in combination with TKIs in the anaplastic thyroid cancer setting [62]. Relatedly, alternating TKI therapy with sorafenib and lenvatinib has shown promise in preclinical work, including in RAI-R DTC cell lines [63], and may warrant clinical exploration.

Supplementary Table S1 summarizes selected ongoing studies of interest regarding TKI therapy of RAI-R DTC.

\section{Recent developments: selective TKIs}

Recently, specific molecular targets have moved into greater focus in the systemic therapy of RAI-R DTC. The August 2020 NCCN guidelines [17] suggest testing for NTRK fusion in patients with "advanced, progressive, or threating” DTC. If such fusion is detected, larotrectinib [64] and entrectinib [65] now have received regulatory approval in Europe as systemic treatment options. However, NTRK fusion is fairly rare in DTC. Similarly, RET also can be specifically targeted; the selective inhibitor selpercatinib [66] now has received US regulatory approval for RET-altered thyroid cancers. 


\section{Conclusions}

A variety of important open issues remain regarding TKI therapy of RAI-R DTC. However, in many cases, the literature and the clinical experience of expert centers provide sufficient data to allow clinicians and patients to undertake reasonable provisional approaches to determining when to start and stop TKIs, which drug to choose as first-line, how to identify net benefit of this form of therapy, and how to manage TKI toxicity. It is hoped that additional research will provide more definitive answers to the key questions and controversies discussed in this paper.

\section{Funding}

Eisai GmbH, Frankfurt, Germany.

\section{Conflict of Interest}

The authors declare the following potential conflicts-of-interest: all authors, honoraria and defrayment of travel and lodging expenses in connection with membership in the Eisai GmbH Advisory Board. Besides the foregoing, the authors declare the following potential conflicts of interest: FAV, consultancy fees from Jubilant Draximage and Sanofi Genzyme, speaker honoraria from Sanofi Genzyme, research support from Eisai; HA, lecture fees from Eisai, GE, Norgine, Novartis, Pfizer, and Sirtex; $I \mathrm{Br}$, support from Bayer AG, Eisai $\mathrm{GmbH}$, and ROTOP Pharma $\mathrm{GmbH}$; $A B$, none; $A D$, none; $C D$, none; $C K$, honoraria for presentation, Novartis, advisory board membership, Eisai, Ipsen, Merck, travel support, Ipsen, Medac; UK, none; MCK, honoraria from Eisai, AstraZeneca, Bayer, Exelixis, GE Healthcare, Lilly, and Sanofi Genzyme, advisory board membership, Eisai, Exelixis, travel support, Eisai, Exelixis, GE Healthcare, Ipsen, Novartis; ML, research support from Exelixis; CR, none; KS, none; HSW, none; AZ, travel assistance from Eisai, honoraria, travel assistance, and advisory board membership, Johnson \& Johnson, research support from Future Diagnostics, MSD, Shire, travel assistance from Gesellschaft für Qualitätssicherung Hessen, stock ownership in Fresenius; MS, none;. Eisai $\mathrm{GmbH}$, Frankfurt, Germany supported the expert panel meetings the content of which is summarized in this paper. Eisai $\mathrm{GmbH}$ also provided medical writing support in the development of this manuscript, and defrayed journal page charges for the accepted manuscript. Robert J. Marlowe, ISMPP CMPPTM, Spencer-Fontayne Corporation, Jersey City, NJ helped draft, edit, and organize the manuscript, and partly handled manuscript submission logistics.

\section{References}

[1] Luster M, Aktolun C, Amendoeira I et al. European perspective on 2015 American Thyroid Association Management Guidelines for Adult Patients with Thyroid Nodules and Differentiated Thyroid Cancer: proceedings of an interactive international Symposium. Thyroid 2019; 29: 7-26

[2] Van Nostrand D. Radioiodine refractory differentiated thyroid cancer: time to update the classifications. Thyroid 2018; 28: 1083-1093

[3] Haugen BR, Alexander EK, Bible KC et al. 2015 American Thyroid Association Management Guidelines for Adult Patients with Thyroid Nodules and Differentiated Thyroid Cancer: The American Thyroid Association Guidelines Task Force on Thyroid Nodules and Differentiated Thyroid Cancer. Thyroid 2016; 26:: 1-133
[4] Gild ML, Topliss DJ, Learoyd D et al. Clinical guidance for radioiodine refractory differentiated thyroid cancer. Clin Endocrinol 2018; 88: 529-537

[5] Berdelou A, Lamartina L, Klain M et al. Treatment of refractory thyroid cancer. Endocr Relat Cancer 2018; 25: R209-R223

[6] Gosain R, Alexander JS, Gill A et al. Radioactive iodine-refractory differentiated thyroid cancer in the elderly. Curr Oncol Rep 2018; 20: 82

[7] Brose MS, Nutting CM, Jarzab B et al. Sorafenib in radioactive iodine-refractory, locally advanced or metastatic differentiated thyroid cancer: a randomised, double-blind, phase 3 trial. Lancet 2014; 384: 319-328

[8] Schlumberger M, Tahara M, Wirth L] et al. Lenvatinib versus placebo in radioiodine-refractory thyroid cancer. New Engl J Med 2015; 372: 621-630

[9] Brose MS, Worden FP, Newbold KL et al. Effect of age on the efficacy and safety of lenvatinib in radioiodine-refractory differentiated thyroid cancer in the Phase III SELECT Trial. J Clin Oncol 2017; 35: 2692-2699

[10] Eisai Inc.. Lenvima (lenvatinib) Summary of Product Characteristics

[11] Bayer Healthcare. Nexavar (sorafenib) Summary of Product Characteristics

[12] Haddad RI, Schlumberger M, Wirth LJ et al. Incidence and timing of common adverse events in lenvatinib-treated patients from the SELECT trial and their association with survival outcomes. Endocrine 2017; 56: 121-128

[13] Yu ST, Ge JN, Luo JY et al. Treatment-related adverse effects with TKIs in patients with advanced or radioiodine refractory differentiated thyroid carcinoma: A systematic review and meta-analysis. Cancer Manag Res 2019; 11: 1525-1532

[14] Giani C, Valerio L, Bongiovanni A et al. Safety and quality of life data from an Italian expanded access program of lenvatinib for treatment of thyroid cancer. Thyroid. 2020, doi:10.1089/thy.2020.0276

[15] Tuttle RM, Brose MS, Grande E et al. Novel concepts for initiating multitargeted kinase inhibitors in radioactive iodine refractory differentiated thyroid cancer. Best Pract Res Clin Endocrinol Metab 2017; 31: 295-305

[16] Masaki C, Sugino K, Saito $N$ et al. Efficacy and limitations of lenvatinib therapy for radioiodine-refractory differentiated thyroid cancer: real-world experiences. Thyroid 2020; 30: 214-221

[17] National Comprehensive Cancer Network. NCCN Clinical Practice Guidelines in Oncology: Thyroid Carcinoma (NCCN Evidence Blocks. Version 2.2020 - 7 August 2020)

[18] KreissI MC, Janssen MJR, Nagarajah J.. Current treatment strategies in metastasized differentiated. thyroid cancer. J Nucl Med 2019; 60: 9-15

[19] Eisenhauer EA, Therasse P, Bogaerts J et al. New response evaluation criteria in solid tumours: revised RECIST guideline (version 1.1). Eur J Cancer 2009; 45: 228-247

[20] Jaber T, Waguespack SG, Cabanillas ME et al. Targeted therapy in advanced. thyroid cancer to resensitize tumors to radioactive iodine. J Clin Endocrinol Metab 2018; 103: 3698-3705

[21] Manohar PM, Beesley L], Bellile EL et al. Prognostic value of FDG-PET/ CT metabolic parameters in metastatic radioiodine-refractory differentiated thyroid cancer. Clin Nucl Med 2018; 43: 641-647

[22] Oken MM, Creech RH, Tormey DC et al. Toxicity and response criteria of the Eastern Cooperative Oncology Group Am J Clin Oncol 1982; 5: 649-655

[23] Berdelou A, Borget I, Godbert Y et al. Lenvatinib for the treatment of radioiodine-refractory. thyroid cancer in real-life practice. Thyroid 2018; $28: 72-78$

[24] Locati LD, Piovesan A, Durante C et al. Real-world efficacy and safety of lenvatinib: data from a compassionate use in the treatment of radioactive iodine-refractory differentiated thyroid cancer patients in Italy. Eur J Cancer 2019; 118: 35-40 
[25] Jerkovich F, Califano I, Bueno F et al. Real-life use of lenvatinib in patients with differentiated thyroid cancer: experience from Argentina. Endocrine 2020; 69: 142-148

[26] Kish JK, Chatterjee D, Wan Y et al. Lenvatinib and subsequent therapy for radioactive iodine-refractory differentiated thyroid cancer: A real-world study of clinical effectiveness in the United States. Adv Ther 2020; 37: 2841-2852

[27] De Leo S, Di Stefano M, Persani L et al. Lenvatinib as first-line treatment for advanced thyroid cancer: long progression-free survival. Endocrine 2020, doi:10.1007/s12020-020-02477-0

[28] Okamoto K, Ikemori-Kawada M, Jestel A et al. Distinct binding mode of multikinase inhibitor lenvatinib revealed by biochemical characterization. ACS Med Chem Lett 2015; 6: 89-94

[29] Tsoli M, Alexandraki KI, Spei ME et al. Anti-tumor activity and safety of multikinase inhibitors in advanced and/or metastatic thyroid cancer: a systematic review and network meta-analysis of randomized controlled trials. Horm Metab Res 2020; 52: 25-31

[30] Schlumberger M, Tahara M, Wirth LJ. Lenvatinib in radioiodine-refractory thyroid cancer. New Engl J Med 2015; 372: 1868

[31] Robinson B, Schlumberger M, Wirth L] et al. Characterization of tumor size changes over time from the phase 3 study of lenvatinib in thyroid cancer. J Clin Endocrinol Metab 2016; 101: 4103-4109

[32] Tahara M, Brose MS, Wirth LJ et al. Impact of dose interruption on the efficacy of lenvatinib in a phase 3 study in patients with radioiodine-refractory differentiated thyroid cancer. Eur J Cancer 2019; 106: 61-68

[33] Giani C, Valerio L, Pieruzzi L et al. Hypertension, nausea, anorexia, and weight loss were significantly associated with better progression-free survival in patients with radioiodine-refractory differentiated thyroid carcinoma treated with lenvatinib. European Society of Medical Oncology, 19-23 October 2018, Munich, Germany

[34] Wirth L], Tahara M, Robinson B et al. Treatment-emergent hypertension and efficacy in the phase 3 Study of (E7080) lenvatinib in differentiated cancer of the thyroid (SELECT). Cancer 2018; 124: 2365-2372

[35] Resteghini C, Cavalieri S, Galbiati D et al. Management of tyrosine kinase inhibitors (TKI) side effects in differentiated and medullary thyroid cancer patients. Best Pract Res Clin Endocrinol Metab 2017; 31: 349-361

[36] Resteghini C, Locati LD, Bossi P et al. Do not throw the baby out with the bathwater: SELECT a personalized, de-escalated lenvatinib schedule allows response in locally advanced DTC while controlling major drug-related bleeding. Ann Oncol 2017; 28: 2321-2322

[37] Dadu R, Waguespack SG, Sherman SI et al. Efficacy and tolerability of different starting doses of sorafenib in patients with differentiated thyroid cancer. Oncologist 2014; 19: 477-482

[38] Chen L, Shen Y, Luo Q et al. Response to sorafenib at a low dose in patients with radioiodine-refractory pulmonary metastases from papillary thyroid carcinoma. Thyroid 2011; 21: 119-124

[39] Lin CY, Chang JS, Huang SM et al. Experience of sorafenib treatment in differentiated thyroid cancer from Taiwan. J Formos Med Assoc 2020; 120: 189-195. DOI: 10.1016/j.jfma.2020.04.021

[40] Ogawa-Morita T, Sano Y, Okano T et al. Validation of a liquid chromatography-tandem mass spectrometric assay for quantitative analysis of lenvatinib in human plasma. Int J Anal Chem 2017; 2017: 2341876

[41] Watanabe Y, Doki K, Sekine I et al. High-performance liquid chromatography for therapeutic drug monitoring of serum lenvatinib. Ther Drug Monit 2020; 42: 554-558

[42] Nagahama M, Ozeki T, Suzuki A et al. Association of lenvatinib trough plasma concentrations with lenvatinib-induced toxicities in Japanese patients with thyroid cancer. Med Oncol 2019; $36: 39$

[43] Tamai T, Hayato $S$, Hojo $S$ et al. Dose finding of lenvatinib in subjects with advanced hepatocellular carcinoma based on population pharmacokinetic and exposure-response analyses. J Clin Pharmacol 2017; 57: 1138-1147
[44] Bellesoeur A, Carton E, Mir O et al. Critical role of sorafenib exposure over time for its antitumor activity in thyroid cancer. Invest New Drugs 2014; 32: 569-572

[45] Jerkovich F, Garcia Falcone MG, Pitoia F.. The experience of an Endocrinology Division on the use of tyrosine multikinase inhibitor therapy in patients with radioiodine-resistant differentiated thyroid cancer. Endocrine 2019; 64: 632-638

[46] Tori M, Shimo T. Long-term efficacy of lenvatinib for recurrent papillary thyroid carcinoma after multimodal treatment and management of complications: A case report. BMC Cancer 2018; 18: 698

[47] Dadu R, Devine C, Hernandez M et al. Role of salvage targeted therapy in differentiated thyroid cancer patients who failed first-line sorafenib. J Clin Endocrinol Metab 2014; 99: 2086-2094

[48] Takinami M, Yokota T. Rechallenge with lenvatinib after refractoriness to initial lenvatinib followed by sorafenib in a patient with metastatic papillary thyroid carcinoma. Case Rep Oncol 2020; 13: 522-527

[49] Huillard O, Jouinot A, Tlemsani C et al. Body composition in patients with radioactive iodine-refractory, advanced differentiated thyroid cancer treated with sorafenib or placebo: A retrospective analysis of the Phase III DECISION Trial. Thyroid 2019; 29: 1820-1827

[50] Capdevila ], Newbold K, Licitra L et al. Optimisation of treatment with lenvatinib in radioactive iodine-refractory differentiated thyroid cancer. Cancer Treat Rev 2018; 69: 164-176

[51] Cabanillas ME, Takahashi S. Managing the adverse events associated with lenvatinib therapy in radioiodine-refractory differentiated thyroid cancer. Sem Oncol 2019; 46: 57-64

[52] Kiess AP, Agrawal N, Brierley JD et al. External-beam radiotherapy for differentiated thyroid cancer locoregional control: A statement of the American Head and Neck Society. Head Neck 2016; 38: 493-498

[53] Kiyota N, Schlumberger M, Muro K et al. Subgroup analysis of Japanese patients in a phase 3 study of lenvatinib in radioiodine-refractory differentiated thyroid cancer. Cancer Sci 2015; 106: 1714-1721

[54] Carhill AA, Cabanillas ME, Jimenez $C$ et al. The noninvestigational use of tyrosine kinase inhibitors in thyroid cancer: establishing a standard for patient safety and monitoring. J Clin Endocrinol Metab 2013; 98: 31-42

[55] Suzuki S, Horinouchi A, Uozumi S et al. Impact of outpatient pharmacy interventions on management of thyroid patients receiving lenvatinib. SAGE Open Med 2020; 8: 2050312120930906

[56] Agrawal VR, Jodon G, Mushtaq R et al. Update on multikinase inhibitor therapy for differentiated thyroid cancer. Drugs Today (Barc) 2018; 54: 535-545

[57] Escudier B, Worden F, Kudo M. Sorafenib: Key lessons from over 10 years of experience. Expert Rev Anticancer Ther 2019; 19: 177-189

[58] Di Desidero T, Orlandi P, Gentile D et al. Pharmacological effects of vinorelbine in combination with lenvatinib in anaplastic thyroid cancer. Pharmacol Res 2020; 158: 104920

[59] Garcia-Rodriguez S, Pinillos GM, Chaves-Conde M. Lenvatininb as treatment for naive patients with aggressive thyroid cancer bone metastases and bad performance status. Case Rep Oncol Med 2020; 2020: 8679149

[60] Dadu R, Shah K, Busaidy NL et al. Efficacy and tolerability of vemurafenib in patients with BRAF(V600E) -positive papillary thyroid cancer: M.D. Anderson Cancer Center off-label experience. J Clin Endocrinol Metab 2015; 100: E77-81

[61] Iva J, Filip G, Martin B et al. The significance of BRAFV600E mutation in thyroid cancer in terms of novel targeted therapies - overview of current knowledge and studies. Klin Onkol 2019; 31: 339-344

[62] Iyer PC, Dadu R, Gule-Monroe M et al. Salvage pembrolizumab added to kinase inhibitor therapy for the treatment of anaplastic thyroid carcinoma. J Immunother Cancer 2018; 6: 68

[63] Kim SY, Kim SM, Chang HJ et al. SoLAT (Sorafenib Lenvatinib alternating treatment): A new treatment protocol with alternating sorafenib and lenvatinib for refractory thyroid cancer. BMC Cancer 2018; 18: 956 
[64] Hong DS, DuBois SG, Kummar S et al. Larotrectinib in patients with TRK fusion-positive solid tumours: A pooled analysis of three phase 1/2 clinical trials. Lancet Oncol 2020; 21: 531-540

[65] Chu YH, Dias-Santagata D, Farahani AA et al. Clinicopathologic and molecular characterization of NTRK-rearranged thyroid carcinoma (NRTC). Mod Pathol 2020; 33: 2189-2197

[66] Wirth L], Sherman E, Robinson B et al. Efficacy of selpercatinib in RET-altered thyroid cancers. New Engl J Med 2020; 383: 825-835

[67] Brose MS, Nutting CM, Sherman SI et al. Rationale and design of DECISION: a double-blind, randomized, placebo-controlled phase III trial evaluating the efficacy and safety of sorafenib in patients with locally advanced or metastatic radioactive iodine (RAI)-refractory, differentiated thyroid cancer. BMC Cancer 2011; 11: 349
[68] Dittus KL, Gramling RE, Ades PA.. Exercise interventions for individuals with advanced cancer: a systematic review. Prev Med 2017; 104: 124-132

[69] Masaki C, Sugino K, Kobayashi S et al. Urinalysis by combination of the dipstick test and urine protein-creatinine ratio (UPCR) assessment can prevent unnecessary lenvatinib interruption in patients with thyroid cancer. Int J Clin Oncol 2020; 25: 1278-1284 